\title{
DILATATIONS AND EXPONENTS OF QUASISYMMETRIC HOMEOMORPHISMS
}

\author{
Tao Cheng and Shanshuang Yang \\ East China Normal University, Department of Mathematics \\ Shanghai, 200241, P. R. China; tcheng@math.ecnu.edu.cn \\ Emory University, Department of Mathematics and Computer Sciences \\ Atlanta, GA 30322, U.S.A.; syang@mathcs.emory.edu
}

\begin{abstract}
Given a quasisymmetric homeomorphism, we introduce the concept of quasisymmetric exponent and explore its relations to other conformal invariants. As a consequence, we establish a necessary and sufficient condition on the equivalence of the dilatation and the maximal dilatation of a quasisymmetric homeomorphism by using the quasisymmetric exponent. A classification on the elements of the universal Teichmüller space is obtained by using this necessary and sufficient condition.
\end{abstract}

\section{Introduction}

Throughout this paper we let $\mathbf{R}$ denote the real line, $\overline{\mathbf{R}}$ its one point compactification $\mathbf{R} \cup\{\infty\}$ and $\mathbf{H}$ the upper half plane in $\mathbf{R}^{2}=\mathbf{R} \times \mathbf{R}$. A (sense preserving) homeomorphism $h$ from $\mathbf{R}$ onto itself is called quasisymmetric if there exists a constant $M$ such that

$$
M^{-1} \leq \frac{h(x+t)-h(x)}{h(x)-h(x-t)} \leq M
$$

for all numbers $x \in \mathbf{R}$ and $t>0$. The above inequality is often called Ahlfors' $M$ condition. It is well known that $h$ is quasisymmetric if and only if it is the boundary value of a quasiconformal mapping of $\mathbf{H}$ onto itself. Furthermore, $h$ is linear if and only if it is the boundary value of a conformal (Möbius) map of $\mathbf{H}$. In other words, a homeomorphism $h$ is quasisymmetric if and only if it has a quasiconformal extension to the upper half plane. This extendability induces the fact that the collection of all quasisymmetric homeomorphisms of $\mathbf{R}$ onto itself form a group. This feature makes the notion of quasisymmetry very useful in the theory of Riemann surfaces as well as in the study of one dimensional complex dynamical systems.

In order to quantify the quasisymmetry of a homeomorphism, several conformal invariants have been introduced. It has been an interesting and important problem for more than fifty years to investigate the relationship between the dilatation $M_{h}$ and maximal dilatation $K_{h}$ (see definitions below) of a quasisymmetric homeomorphism $h$ of the real line. From the conformal geometry point of view, the dilatation $M_{h}$ measures how much a given quasisymmetric homeomorphism changes the extremal distance between continua on the real line $\mathbf{R}$, while the maximal dilatation $K_{h}$ measures how much an extremal quasiconformal extension of the given quasisymmetric homeomorphism changes the extremal distance between continua in the upper half

doi:10.5186/aasfm.2016.4122

2010 Mathematics Subject Classification: Primary 30C62, 30C70.

Key words: Quasisymmetric homeomorphism, modulus, dilatation, quasisymmetric exponent. The first author is partially supported by NNSF (No. 11371268 and No. 11471117) and STCSM (No. 13dz2260400). The second author is partially supported by NNSF (No. 11471117). 
plane. It was conjectured, informally since the 1960's, that $M_{h}=K_{h}$ for any homeomorphism. However, Anderson and Hinkkanen (see [3]) disproved this conjecture by constructing a concrete example of a family of affine mappings of some parallelograms. Thus, a natural question to ask is under what conditions the equality holds. After Anderson and Hinkkanen's work, many concepts and methods were introduced to investigate the relation between these two quantities. This paper is also devoted to this endeavor.

1.1. Dilatations $\boldsymbol{M}_{\boldsymbol{h}}$ and $\boldsymbol{K}_{\boldsymbol{h}}$. Given a quasisymmetric homeomorphism $h$, in order to quantify its quasisymmetry (or to measure how far it is from being conformal), we define several conformal invariants (called dilatations) for $h$ and study their relations. These dilatations, in one way or the other, measure how much a homeomorphism or its extensions distort the moduli of certain curve families.

For a curve family $\Gamma$ in the plane, its (conformal) modulus, denoted by $\bmod (\Gamma)$, is defined as

$$
\bmod (\Gamma)=\inf \int_{\mathbf{R}^{2}} \rho^{2} d m
$$

where the infimum is taken over the set, denoted by $\operatorname{adm}(\Gamma)$, of all non-negative Borel measurable functions $\rho: \mathbf{R}^{2} \rightarrow \mathbf{R}$ such that $\int_{\gamma} \rho d s \geq 1$ for every locally rectifiable curve $\gamma$ in $\Gamma$. The extremal length $\lambda(\Gamma)$ of a curve family $\Gamma$ is defined as $\lambda(\Gamma)=$ $1 / \bmod (\Gamma)$. The most frequently studied curve family is the one that joins two disjoint continua $A$ and $B$ in a domain $D$, and its modulus is denoted by $\bmod (A, B ; D)$. We refer the reader to [1] and [2] for basic definitions and properties about the modulus and extremal length.

Given an orientation preserving (quasisymmetric) homeomorphism $h$ of $\mathbf{R}$ onto itself, there are two important constants associated with $h$. The first one, denoted by $M_{h}$, is called the dilatation of $h$ and is defined as

$$
M_{h}=\sup \frac{\bmod (h(A), h(B) ; \mathbf{H})}{\bmod (A, B ; \mathbf{H})}
$$

where the supremum is taken over all pairs of disjoint nondegenerate continua $A$ and $B$ on the real line. Another one, denoted by $K_{h}$, is called the maximal dilatation of $h$. Let $Q C(h)$ be the class of all quasiconformal mappings $f$ of the closed upper half plane $\overline{\mathbf{H}}=\mathbf{H} \cup \mathbf{R}$ onto itself with boundary value $h$. The maximal dilatation $K_{h}$ is defined as

$$
K_{h}=\inf \{K(f): f \in Q C(h)\},
$$

where $K(f)$ is the maximal dilatation of a quasiconformal mapping $f \in Q C(h)$ and can be defined as

$$
K(f)=\sup \frac{\bmod (f(\Gamma))}{\bmod (\Gamma)},
$$

where the supremum is taken over all curve families $\Gamma$ in $\mathbf{H}$ such that $\bmod (f(\Gamma))$ and $\bmod (\Gamma)$ are not simultaneously zero or infinity. Clearly, it follows from the definitions that $M_{h^{-1}}=M_{h} \geq 1$ and that $K_{h^{-1}}=K_{h} \geq 1$. It is also easy to observe that $M_{h}=$ $K_{h}=1$ if and only if $h$ is linear (or the boundary value of a Möbius transformation). A quasiconformal extension $f$ of $h$ onto $\mathbf{H}$ is called extremal if $K(f)=K_{h}$. It is well known that there always exists at least one extremal mapping in the class $Q C(h)$ (see $[19,20]$ ). Thus, for a given quasisymmetric homeomorphism $h$, its maximal dilatation $K_{h}$ is just the maximal dilatation of an extremal quasiconformal extension of $h$. This justifies the terminology and notation used here for the quantity $K_{h}$. 
We want to point out that, in some of the existing literature, the quantity $M_{h}$ defined above is called maximal dilatation of $h$ and denoted by $K_{h}$ or $K_{0}(h)$, while the quantity $K_{h}$ defined above has been denoted by $K_{h}^{*}$ or $K^{*}(h)$. The purpose of introducing the new names and new notation here is to give more intuitive terms and notation for these quantities. One should also note that the dilatation $M_{h}$ can be defined in terms of moduli of quadrilaterals with domain $\mathbf{H}$ and vertices on the real line (see [3] and [24]).

1.2. Boundary dilatation. A quasisymmetric homeomorphism $h$ from $\mathbf{R}$ onto itself also determines another constant which is called the boundary dilatation of $h$ (see [21] and [22]). The local boundary dilatation of $h$ at a point $\zeta \in \mathbf{R}$ is defined as:

$$
H_{h}(\zeta)=\inf _{f}\{K(f): f \text { is a } \mathrm{QC} \text { extension of } h \text { in a neighborhood of } \zeta\},
$$

where the infimum is taken over all possible quasiconformal extensions $f$ of $h$ to neighborhoods of $\zeta$. The boundary dilatation of $h$ is then defined as

$$
H_{h}=\sup _{\zeta \in \mathbf{R}} H_{h}(\zeta)
$$

It is easy to see that $H_{h^{-1}}=H_{h}$. Also, as Fehlmann (see [8]) pointed out, the supremum in the above definition is achieved, that is,

$$
H_{h}=\max _{\zeta \in \overline{\mathbf{R}}} H_{h}(\zeta)
$$

1.3. Relations among the dilatations. Obviously, the above defined constants associated with a quasisymmetric homeomorphism $h$ are all invariant under Möbius transformations, and hence are often referred to as conformal invariants. It is easy to see that they satisfy the following inequalities.

$$
H_{h} \leq K_{h}, \quad M_{h} \leq K_{h} .
$$

However, the relationship between $H_{h}$ and $M_{h}$ is not clear.

It had been a long standing open question whether the conjectured relation $M_{h}=K_{h}$ always holds for any quasisymmetric homeomorphism, until Anderson and Hinkkanen [3] constructed an example disproving this conjecture. Later, $\mathrm{Wu}$ [24] and Yang [26] independently established a necessary condition such that $M_{h}=K_{h}$. In order to state their result, we need the following definitions.

A point $\zeta \in \mathbf{R}$ is called a substantial boundary point of $h$ if $H_{h}(\zeta)=K_{h}$, meaning that $h$ cannot be extended to any neighborhood of $\zeta$ without reaching the global maximal dilatation $K_{h}$. A quasisymmetric homeomorphism $h$ of $\mathbf{R}$ onto itself is said to be induced by an affine mapping if it is the restriction to $\mathbf{R}$ of a map of the form $\phi_{2} \circ A_{K} \circ \phi_{1}$, where $A_{K}(x+i y)=x+i K y$ is an affine map, while $\phi_{1}$ and $\phi_{2}$ are conformal mappings from a rectangle $\{x+i y: 0<x<a, 0<y<b\}$ and its image $\{u+i v: 0<u<a, 0<v<K b\}$ under $A_{K}$ onto $\mathbf{H}$, respectively. The necessary condition for $M_{h}=K_{h}$ established by Wu (see [24]) and Yang (see [26]) can be stated as follows.

Theorem A. [24, 26] Let $h: \mathbf{R} \rightarrow \mathbf{R}$ be a quasisymmetric homeomorphism. If $M_{h}=K_{h}$, then either $h$ is induced by an affine mapping or $H_{h}=K_{h}$ (that is, $h$ has a substantial boundary point).

In [24] and [26], both authors asked whether the necessary condition is also sufficient. Shiga and Tanigawa [18] gave an implicit counterexample by proving the existence of a homeomorphism $h$ for which $H_{h}=K_{h}$ and $M_{h}<K_{h}$. Later, Shen [16] proved that there exists a family of quasisymmetric homeomorphisms $h$ such that 
$M_{h}<K_{h}=H_{h}$ by analyzing a concrete example constructed by Strebel. From a totally different perspective, J. Chen and Z. Chen [5] gave a necessary and sufficient condition for the equality $M_{h}=K_{h}$ by using the method of quadratic differentials and the main inequality (see [15]). This result can be stated as follows.

Theorem B. [5] Let $h$ be a quasisymmetric homeomorphism of $\mathbf{R}$ and let $f(z)$ be an extremal quasiconformal extension of $h$ to the upper half plane $\mathbf{H}$ with complex dilatation $\mu(z)$. Then the equality $M_{h}=K_{h}$ holds if and only if

$$
\sup _{Q} \operatorname{Re} \iint_{\mathbf{H}} \mu(z) \Phi_{Q}^{\prime 2}(z) d x d y=\|\mu\|_{\infty}
$$

where the supremum is taken over all quadrilaterals $Q=Q\left(z_{1}, z_{2}, z_{3}, z_{4}\right)$ with $\mathbf{H}$ as its domain and vertices $z_{1}, z_{2}, z_{3}, z_{4} \in \mathbf{R}$ and $\Phi_{Q}(z)$ maps $Q$ conformally onto a rectangle

$$
R=\{\zeta=\xi+i \eta: 0 \leq \xi \leq a, 0 \leq \eta \leq b, a b=1\} .
$$

In a special case, Strebel (see [19]), from a geometric point of view, gave the following necessary and sufficient condition: if $h$ has no substantial boundary point, then $M_{h}=K_{h}$ if and only if $h$ is induced by an affine mapping.

Therefore, to completely solve the converse problem of Theorem A, one needs to find a necessary and sufficient condition for $M_{h}=K_{h}$ when $h$ has a substantial boundary point. The main purpose of this paper is to do just that. For this we need to introduce a key ingredient called quasisymmetric exponent $\alpha_{h}$ (see Section 2 for definition).

1.4. Summary. One of our main results (Theorem 1) says that for a given quasisymmetric homeomorphism $h$ of the real line $\mathbf{R}$ onto itself, we always have $\alpha_{h} \leq H_{h} \leq K_{h}$ and $\alpha_{h} \leq M_{h} \leq K_{h}$. That means the quasisymmetric exponent can serve as a common lower bound for these three different dilatations. Based on this fundamental result, we give a necessary and sufficient condition (Theorem 2) for the dilatation of a quasisymmetric homeomorphism to be equal to its maximal dilatation. That is, $M_{h}=K_{h}$ if and only if either $\alpha_{h}=K_{h}$ or $h$ is induced by an affine mapping. A classification of elements in the universal Teichmüller space can be obtained by using this necessary and sufficient condition (Theorem 3). Furthermore, we also explore some connection between the quasiextremal distance (QED) constant and the quasisymmetric exponent.

This paper is organized as follows. In Section 2, we introduce the concept of quasisymmetric exponent $\alpha_{h}$ for a quasisymmetric homeomorphism. In Section 3, we compare various conformal invariants and show that the quasisymmtric exponent is the smallest among all of them. Section 4 is devoted to the proof of a necessary and sufficient condition for the equality $M_{h}=K_{h}$ and its corollaries. In Section 5 some further applications of the main results will be given. One is to establish a relation between the quasiconformal reflection constant and the quasiextremal distance (or QED) constant of a Jordan domain in the plane. Another is to give a classification of all quasisymmetric homeomorphisms and the elements in the universal Teichmüller space.

\section{Quasisymmetric exponent}

Recall that quasisymmetric homeomorphisms were introduced by Beurling and Ahlfors [4] as the boundary values of quasiconformal self mappings of the upper half plane. They showed that a homeomorphism of $\mathbf{R}$ is quasisymmetric if and only 
if it satisfies the well known $M$-condition. Later, this very important concept was extended to embeddings in Euclidean spaces and more general metric spaces (see, for example, $[11,23])$. To motivate the concept of quasisymmetric exponent, we recall the following general definition and basic properties for quasisymmetric maps.

Note that the quasisymmetry of a homeomorphism of $\mathbf{R}$ is traditionally defined by using Ahlfors' $M$-condition. In the general metric space setting, following Tukia and Väisälä [23], an embedding $h: X \rightarrow Y$ (in metric spaces) is called quasisymmetric (or QS), if there is a homeomorphism $\eta:[0, \infty) \rightarrow[0, \infty)$ such that

$$
\frac{|c-b|}{|b-a|} \leq t \Longrightarrow \frac{|h(c)-h(b)|}{|h(b)-h(a)|} \leq \eta(t)
$$

for all distinct points $a, b, c \in X$ and for all $t>0$. In this case we also say $f$ is $\eta$-QS. As proved by Tukia and Väisälä [23], these two definitions are equivalent in $\mathbf{R}$. From the definition of $\eta$-QS, $h$ is quasisymmetric if it distorts relative distances by a bounded amount controlled by the distortion function $\eta$. It is well known that, in the Euclidean space setting, one can always take the distortion function in the following special form (see [11, 23]):

$$
\eta(t)=C \max \left\{t^{\lambda}, t^{1 / \lambda}\right\}
$$

where $C \geq 1$ and $\lambda \geq 1$ are constants depending only on the quasisymmetric data of $h$ (namely, the original distortion function).

Thus, to quantify the quasisymmetry of a quasisymmetric homeomorphism, it is natural for us to introduce the concept of quasisymmetric exponent as follows.

Definition 1. Suppose $h$ is a quasisymmetric homeomorphism of $\mathbf{R}$ onto itself. For any given $x \in \mathbf{R}$, the local quasisymmetric exponent of $h$ at $x$, denoted by $\alpha_{h}(x)$, is defined as

$$
\alpha_{h}(x)=\inf \lambda
$$

where the infimum is taken over all exponent $\lambda \geq 1$ such that there exist constant $M$ and a neighborhood $N$ of $x$ with the property that

$$
\frac{|c-b|}{|b-a|} \leq t \Longrightarrow \frac{|h(c)-h(b)|}{|h(b)-h(a)|} \leq M \max \left\{t^{\lambda}, t^{\frac{1}{\lambda}}\right\}
$$

for all distinct triples $a, b, c \in N$. Furthermore, the quasisymmetric exponent of $h$ is defined as

$$
\alpha_{h}=\sup _{x \in \mathbf{R}} \alpha_{h}(x) .
$$

Note that, like the other constants with respect to a quasisymmetric homeomorphism $h$, the quasisymmetric exponents $\alpha_{h}(x)$ and $\alpha_{h}$ are also invariant under Möbius transformations. In this paper, we establish some fundamental relations among these constants.

Before proceeding to the main results, we want to point out one major advantage of the quasisymmetric exponent. It is local and easy to estimate without using quasiconformal extensions or moduli of curve families. As a result, many existing counterexamples are easy consequences of our main results.

\section{Comparison between the quasisymmetric exponent and dilatations}

In this section, we will focus on establishing some fundamental relations among the four constants $\alpha_{h}, H_{h}, M_{h}$ and $K_{h}$ for any given quasisymmetric homeomorphism $h$. In particular, we show that the quasisymmetric exponent $\alpha_{h}$ is the smallest among 
these invariants. The common ground for such comparisons lies in the fact, which will be established here, that these constants dictate the change of cross-ratios under $h$ in a similar fashion.

3.1. The Teichmüller function $\Psi(t)$. To estimate the moduli of certain curve families, the Teichmüller function $\Psi(t)$ associated with the Teichmüller ring plays an important role. Here we state its definition and some basic properties. More details can be found in [1]. Recall that for any domain $D$ and two disjoint nondegenerate continua $A$ and $B$ in $\bar{D}$, we let $\bmod (A, B ; D)$ denote the conformal modulus of the curve family joining $A$ and $B$ in $D$. The Teichmüller function $\Psi(t)$ $(t>0)$ is determined by

$$
\bmod ([-1,0],[t, \infty] ; \mathbf{C})=\frac{2 \pi}{\ln \Psi(t)},
$$

where $[a, b]$ denotes the line segment joining $a$ and $b$. It is well known that $\Psi(t)$ is strictly increasing and that

$$
\lim _{t \rightarrow \infty} \frac{\Psi(t)}{t}=16, \quad \lim _{t \rightarrow \infty} \frac{\ln \Psi(t)}{\ln t}=1, \quad \lim _{t \rightarrow 0} \frac{2 \pi}{\ln \Psi(t)}=\infty .
$$

These limits will be used frequently without mentioning in this paper.

3.2. Change of cross-ratios under $\boldsymbol{h}$. In order to compare the quasisymmetric exponent with the dilatations $M_{h}$ and $H_{h}$, we need the following preliminary results which are also interesting on their own. They exhibit how these constants dictate the change of cross-ratios under a homeomorphism. In what follows, we let $h$ be a quasisymmetric homeomorphism of $\mathbf{R}$. For any point $a \in \mathbf{R}$ its image under $h$ will be denoted by $a^{\prime}$. The cross-ratio of four distinct points $a, b, c, d \in \mathbf{R}$ is defined as

$$
[a, b, c, d]=\frac{|c-b||d-a|}{|b-a||d-c|}
$$

Conventionally, we shall always pass to subsequences if necessary to make the limits involved exist (finite or infinite).

Lemma 1. Let $h$ be a quasisymmetric homeomorphism of $\mathbf{R}$ and let $a_{n}<b_{n}<c_{n}$ be sequences of points in $\mathbf{R}$ all converging to the origin with $\tau_{n}=\left|c_{n}-b_{n}\right| /\left|b_{n}-a_{n}\right| \rightarrow$ $\infty$ or 0 as $n \rightarrow \infty$. Then, for $\tau_{n}^{\prime}=\left|c_{n}^{\prime}-b_{n}^{\prime}\right| /\left|b_{n}^{\prime}-a_{n}^{\prime}\right|$, we have

$$
\frac{1}{\alpha_{h}} \leq \lim _{n \rightarrow \infty} \frac{\ln \tau_{n}}{\ln \tau_{n}^{\prime}} \leq \alpha_{h}
$$

and

$$
\frac{1}{H_{h}} \leq \lim _{n \rightarrow \infty} \frac{\ln \tau_{n}}{\ln \tau_{n}^{\prime}} \leq H_{h}, \quad \frac{1}{M_{h}} \leq \lim _{n \rightarrow \infty} \frac{\ln \tau_{n}}{\ln \tau_{n}^{\prime}} \leq M_{h} .
$$

Proof. Since the constants $\alpha_{h}, H_{h}, M_{h}$ are defined from totally different perspectives, these inequalities need to be treated differently as well. For the proof of (1), by considering $1 / \tau_{n}$ if needed, we may assume that $\tau_{n}=\left|c_{n}-b_{n}\right| /\left|b_{n}-a_{n}\right| \rightarrow \infty$. For a fixed $\varepsilon>0$, by the definition of $\alpha_{h}$, there exists a constant $M<\infty$ such that

$$
\frac{1}{\tau_{n}^{\prime}} \leq M\left(\frac{1}{\tau_{n}}\right)^{\frac{1}{\alpha_{h}+\varepsilon}}
$$


for sufficiently large $n$. Thus it follows that

$$
\lim _{n \rightarrow \infty} \frac{\ln \tau_{n}}{\ln \tau_{n}^{\prime}} \leq \lim _{n \rightarrow \infty} \frac{\ln \frac{1}{\tau_{n}}}{\ln M+\frac{1}{\alpha_{h}+\varepsilon} \ln \frac{1}{\tau_{n}}}=\alpha_{h}+\varepsilon .
$$

On the other hand,

$$
\tau_{n}^{\prime} \leq M \tau_{n}^{\alpha_{h}+\varepsilon} \Longrightarrow \lim _{n \rightarrow \infty} \frac{\ln \tau_{n}^{\prime}}{\ln \tau_{n}} \leq \alpha_{h}+\varepsilon
$$

Thus, letting $\varepsilon \rightarrow 0$ yields the desired inequalities for $\alpha_{h}$.

For the proof of the inequalities for $H_{h}$, first assume that $\tau_{n} \rightarrow 0$. Choose a sequence $d_{n} \rightarrow 0$ such that

$$
\lim _{n \rightarrow \infty}\left|\frac{d_{n}-a_{n}}{d_{n}-c_{n}}\right|=1, \quad \lim _{n \rightarrow \infty} \frac{\left|d_{n}\right|}{\max \left\{\left|a_{n}\right|,\left|b_{n}\right|,\left|c_{n}\right|\right\}}=\infty .
$$

For example, it is easy to verify that the sequence

$$
d_{n}=\sqrt{\max \left\{\left|a_{n}\right|,\left|b_{n}\right|,\left|c_{n}\right|\right\}}
$$

has the desired properties. Let $d_{n}^{\prime}=h\left(d_{n}\right)$. Since $h$ is a quasisymmetric homeomorphism with $h(0)=0$, it follows that

$$
\lim _{n \rightarrow \infty} d_{n}^{\prime}=0, \quad \lim _{n \rightarrow \infty} \frac{\left|d_{n}^{\prime}\right|}{\max \left\{\left|a_{n}^{\prime}\right|,\left|b_{n}^{\prime}\right|,\left|c_{n}^{\prime}\right|\right\}}=\infty .
$$

Furthermore, by quasisymmetry again,

$$
\lim _{n \rightarrow \infty}\left|\frac{c_{n}-a_{n}}{d_{n}-c_{n}}\right|=0 \Longrightarrow \lim _{n \rightarrow \infty}\left|\frac{c_{n}^{\prime}-a_{n}^{\prime}}{d_{n}^{\prime}-c_{n}^{\prime}}\right|=0 .
$$

Thus, it follows that

$$
\lim _{n \rightarrow \infty}\left|\frac{d_{n}^{\prime}-a_{n}^{\prime}}{d_{n}^{\prime}-c_{n}^{\prime}}\right|=1
$$

We shall derive the desired inequalities in (2) by using various modulus estimates. To this end, we denote the line segments $\left[a_{n}, b_{n}\right]$ and $\left[c_{n}, d_{n}\right]$ by $A_{n}$ and $B_{n}$, respectively, and their images under $h$ by $A_{n}^{\prime}$ and $B_{n}^{\prime}$, respectively.

By the definition of the boundary dilatation $H_{h}$, for any fixed $\varepsilon>0$, there exists a neighborhood $U$ of 0 in the complex plane, such that $h$ has a quasiconformal extension $f$ in $U$ whose maximal dilatation in $U$ is less than or equal to $H_{h}+\varepsilon$. Let $U^{\prime}=f(U)$. By the quasi-invariance of modulus, it follows that

$$
\frac{1}{H_{h}+\varepsilon} \leq \frac{\bmod \left(A_{n}^{\prime}, B_{n}^{\prime} ; U^{\prime}\right)}{\bmod \left(A_{n}, B_{n} ; U\right)} \leq H_{h}+\varepsilon
$$

Fix a circular ring $A(0 ; r, R)=\{z: 0<r<|z|<R\}$ in $U$. Since $a_{n}, b_{n}, c_{n}, d_{n} \rightarrow 0$ as $n \rightarrow \infty$, for sufficiently large $n$, each curve joining $A_{n}$ and $B_{n}$ in $\mathbf{C}$ either stays in $U$ or contains a subarc that joins the circles $|z|=r$ and $|z|=R$ in $A(0 ; r, R)$. Thus, using some basic properties of the modulus, one can derive that

$$
\bmod \left(A_{n}, B_{n} ; U\right) \leq \bmod \left(A_{n}, B_{n} ; \mathbf{C}\right) \leq \bmod \left(A_{n}, B_{n} ; U\right)+\frac{2 \pi}{\ln \frac{R}{r}} .
$$

Since

$$
\bmod \left(A_{n}, B_{n} ; \mathbf{C}\right)=\frac{2 \pi}{\ln \Psi\left(\tau_{n}\left|\frac{d_{n}-a_{n}}{d_{n}-c_{n}}\right|\right)} \rightarrow \infty
$$


as $n \rightarrow \infty$, it follows that

$$
\lim _{n \rightarrow \infty} \frac{\bmod \left(A_{n}, B_{n} ; \mathbf{C}\right)}{\bmod \left(A_{n}, B_{n} ; U\right)}=1
$$

Similarly, we have

$$
\lim _{n \rightarrow \infty} \frac{\bmod \left(A_{n}^{\prime}, B_{n}^{\prime} ; \mathbf{C}\right)}{\bmod \left(A_{n}^{\prime}, B_{n}^{\prime} ; U^{\prime}\right)}=1
$$

Therefore, by considering the Teichmüller ring whose complementary components are $A_{n}$ and $B_{n}$ and its conjugate ring, it follows that

$$
\lim _{n \rightarrow \infty} \frac{\bmod \left(A_{n}^{\prime}, B_{n}^{\prime} ; U^{\prime}\right)}{\bmod \left(A_{n}, B_{n} ; U\right)}=\lim _{n \rightarrow \infty} \frac{\bmod \left(A_{n}^{\prime}, B_{n}^{\prime} ; \mathbf{C}\right)}{\bmod \left(A_{n}, B_{n} ; \mathbf{C}\right)}=\lim _{n \rightarrow \infty} \frac{2 \pi / \ln \Psi\left(\frac{1}{\tau_{n}}\right)}{2 \pi / \ln \Psi\left(\frac{1}{\tau_{n}^{\prime}}\right)}=\lim _{n \rightarrow \infty} \frac{\ln \frac{1}{\tau_{n}^{\prime}}}{\ln \frac{1}{\tau_{n}}} .
$$

This together with (3) yields that

$$
\frac{1}{H_{h}+\varepsilon} \leq \lim _{n \rightarrow \infty} \frac{\ln \tau_{n}}{\ln \tau_{n}^{\prime}} \leq H_{h}+\varepsilon
$$

and the first part of (2) follows by letting $\varepsilon \rightarrow 0$.

Next assume that $\tau_{n} \rightarrow \infty$. In this case, we let

$$
\tilde{\tau}_{n}=\frac{1}{\tau_{n}}=\frac{\left|a_{n}-b_{n}\right|}{\left|c_{n}-b_{n}\right|} .
$$

Then $\tilde{\tau}_{n} \rightarrow 0$. With $A_{n}$ and $B_{n}$ being replaced by $A_{n}=\left[-d_{n}, a_{n}\right]$ and $B_{n}=\left[b_{n}, c_{n}\right]$, respectively, the above argument shows that

$$
\frac{1}{H_{h}+\varepsilon} \leq \lim _{n \rightarrow \infty} \frac{\ln \tilde{\tau}_{n}}{\ln \tilde{\tau}_{n}^{\prime}} \leq H_{h}+\varepsilon
$$

which also yields the desired inequalities by letting $\varepsilon \rightarrow 0$.

Finally, we prove the inequalities for $M_{h}$ in (2). Similar to the proof of the inequalities for $H_{h}$ above, we only need to consider the case when $\tau_{n} \rightarrow 0$. For this, we choose the same sequence $\left\{d_{n}\right\}$, and let $A_{n}=\left[a_{n}, b_{n}\right], B_{n}=\left[c_{n}, d_{n}\right], d_{n}^{\prime}=$ $h\left(d_{n}\right), A_{n}^{\prime}=h\left(A_{n}\right)$ and $B_{n}^{\prime}=h\left(B_{n}\right)$. Using some basic properties of the Teichmüller ring and Teichmüller function mentioned above, one deduces that

$$
\lim _{n \rightarrow \infty} \frac{\bmod \left(A_{n}^{\prime}, B_{n}^{\prime} ; \mathbf{C}\right)}{\bmod \left(A_{n}, B_{n} ; \mathbf{C}\right)}=\lim _{n \rightarrow \infty} \frac{2 \pi / \ln \Psi\left(\frac{1}{\tau_{n}}\right)}{2 \pi / \ln \Psi\left(\frac{1}{\tau_{n}^{\prime}}\right)}=\lim _{n \rightarrow \infty} \frac{\ln \frac{1}{\tau_{n}^{\prime}}}{\ln \frac{1}{\tau_{n}}} .
$$

On the other hand, by definition of the dilatation $M_{h}$, it follows that

$$
\frac{1}{M_{h}} \leq \frac{\bmod \left(A_{n}^{\prime}, B_{n}^{\prime} ; \mathbf{C}\right)}{\bmod \left(A_{n}, B_{n} ; \mathbf{C}\right)} \leq M_{h}
$$

Therefore

$$
\frac{1}{M_{h}} \leq \lim _{n \rightarrow \infty} \frac{\ln \frac{1}{\tau_{n}^{\prime}}}{\ln \frac{1}{\tau_{n}}} \leq M_{h}
$$

This completes the proof of Lemma 1.

Note that $\tau_{n}$ can be thought of as the cross-ratio $\left[a_{n}, b_{n}, c_{n}, \infty\right]$ with the fourth point at $\infty$. The next result shows how the constants $\alpha_{h}, H_{h}$ and $M_{h}$ control the change of the cross-ratio of four finite points all converging to the origin. 
Lemma 2. Let $h$ be a quasisymmetric homeomorphism of $\mathbf{R}$ and let $a_{n}<$ $b_{n}<c_{n}<d_{n}$ be sequences of points in $\mathbf{R}$ all converging to the origin with $t_{n}=$ $\left[a_{n}, b_{n}, c_{n}, d_{n}\right] \rightarrow \infty$ or 0 as $n \rightarrow \infty$. Then, for $t_{n}^{\prime}=\left[a_{n}^{\prime}, b_{n}^{\prime}, c_{n}^{\prime}, d_{n}^{\prime}\right]$, we have

$$
\frac{1}{\alpha_{h}} \leq \lim _{n \rightarrow \infty} \frac{\ln t_{n}}{\ln t_{n}^{\prime}} \leq \alpha_{h}
$$

Furthermore, as in Lemma 1, the same inequalities hold with $\alpha_{h}$ being replaced by $H_{h}$ or $M_{h}$.

Proof. Without loss of generality, we may assume $t_{n} \rightarrow \infty$. By switching the roles of $\left[a_{n}, b_{n}\right]$ and $\left[c_{n}, d_{n}\right]$ if needed, we may further assume that $r_{n}=\left|b_{n}-a_{n}\right| /\left|d_{n}-c_{n}\right|$ is bounded. Then

$$
t_{n}=\frac{\left|c_{n}-b_{n}\right|\left|d_{n}-a_{n}\right|}{\left|b_{n}-a_{n}\right|\left|d_{n}-c_{n}\right|}=\tau_{n}\left(1+r_{n}+\sigma_{n}\right),
$$

where

$$
\tau_{n}=\frac{\left|c_{n}-b_{n}\right|}{\left|b_{n}-a_{n}\right|}, \quad \sigma_{n}=\frac{\left|c_{n}-b_{n}\right|}{\left|d_{n}-c_{n}\right|}=r_{n} \tau_{n}
$$

We let $t_{n}^{\prime}, \tau_{n}^{\prime}, \sigma_{n}^{\prime}, r_{n}^{\prime}$ denote the corresponding quantities as determined by $a_{n}^{\prime}, b_{n}^{\prime}, c_{n}^{\prime}, d_{n}^{\prime}$.

Since $r_{n}$ is bounded, by (4) and the assumption that $t_{n} \rightarrow \infty$, it follows that $\tau_{n} \rightarrow \infty$. Thus we only need to consider two cases: $\sigma_{n}$ is bounded or $\sigma_{n} \rightarrow \infty$.

If $\sigma_{n}$ is bounded, $\sigma_{n}^{\prime}$ is also bounded due to the quasisymmetry of $h$. Thus, (4) implies that

$$
\lim _{n \rightarrow \infty} \frac{\ln t_{n}}{\ln t_{n}^{\prime}}=\lim _{n \rightarrow \infty} \frac{\ln \tau_{n}}{\ln \tau_{n}^{\prime}},
$$

which, together with Lemma 1 yields the desired inequalities.

Finally, assume that $\sigma_{n} \rightarrow \infty$. Then, it follows again from (4) that

$$
\frac{t_{n}}{\tau_{n} \sigma_{n}}=\frac{1+r_{n}+\sigma_{n}}{\sigma_{n}} \rightarrow 1
$$

Thus, we have

$$
\lim _{n \rightarrow \infty} \frac{\ln t_{n}}{\ln t_{n}^{\prime}}=\lim _{n \rightarrow \infty} \frac{\ln \tau_{n}+\ln \sigma_{n}}{\ln \tau_{n}^{\prime}+\ln \sigma_{n}^{\prime}} .
$$

It is easy to see that the desired inequalities follow from this and Lemma 1 applied to both $\tau_{n}$ and $\sigma_{n}$.

3.3. Comparison of $\boldsymbol{\alpha}_{\boldsymbol{h}}$ with dilatations. Now we are ready to compare the quasisymmetric exponent $\alpha_{h}$ with the various dilatations $H_{h}, M_{h}$, and $K_{h}$ of $h$. The first result states that the quasisymmetric exponent $\alpha_{h}$ is always a lower bound for the boundary dilatation $H_{h}$.

Proposition 1. For any quasisymmetric homeomorphism $h$ of the real line $\mathbf{R}$ onto itself, $\alpha_{h} \leq H_{h}$.

Proof. By definition of the quasisymmetric exponent $\alpha_{h}$, it suffices to show that for each fixed $x \in \mathbf{R}, \alpha_{h}(x) \leq H_{h}$. By composing with Möbius transformations if necessary, we may assume that $x=0$ and $h(0)=0$. Hence we will focus on the proof of $\alpha_{h}(0) \leq H_{h}$. To this end, we only need to show that, for any given $\varepsilon>0$, there exist a neighborhood $N$ of 0 and a constant $M<\infty$ such that

$$
\left|\frac{c-b}{b-a}\right| \leq t \Longrightarrow\left|\frac{c^{\prime}-b^{\prime}}{b^{\prime}-a^{\prime}}\right| \leq M \max \left\{t^{\frac{1}{H_{h}+\varepsilon}}, t^{H_{h}+\varepsilon}\right\}
$$


for any distinct triplets $a, b, c \in N$, where $a^{\prime}, b^{\prime}, c^{\prime}$ denote the images of $a, b, c$, respectively.

Suppose (5) is not true. Then there exist a constant $\varepsilon>0$ and sequences of points $a_{n}, b_{n}, c_{n} \rightarrow 0$ as $n \rightarrow \infty$ such that

$$
\frac{\left|\frac{c_{n}^{\prime}-b_{n}^{\prime}}{b_{n}^{\prime}-a_{n}^{\prime}}\right|}{\left|\frac{c_{n}-b_{n}}{b_{n}-a_{n}}\right|^{\frac{1}{H_{h}+\varepsilon}}} \rightarrow \infty \text { and } \frac{\left|\frac{c_{n}^{\prime}-b_{n}^{\prime}}{b_{n}^{\prime}-a_{n}^{\prime}}\right|}{\left|\frac{c_{n}-b_{n}}{b_{n}-a_{n}}\right|^{H_{h}+\varepsilon}} \rightarrow \infty
$$

as $n \rightarrow \infty$. Let

$$
\tau_{n}=\left|\frac{c_{n}-b_{n}}{b_{n}-a_{n}}\right|, \quad \tau_{n}^{\prime}=\left|\frac{c_{n}^{\prime}-b_{n}^{\prime}}{b_{n}^{\prime}-a_{n}^{\prime}}\right| .
$$

Taking the logarithm in (6) yields

$$
\frac{1}{H_{h}+\varepsilon} \ln \frac{1}{\tau_{n}}-\ln \frac{1}{\tau_{n}^{\prime}} \rightarrow \infty \quad \text { and } \quad \ln \tau_{n}^{\prime}-\left(H_{h}+\varepsilon\right) \ln \tau_{n} \rightarrow \infty .
$$

In order to derive a contradiction with (7), by passing to subsequences if necessary, we may assume that $\tau_{n} \rightarrow \tau$ and $\tau_{n}^{\prime} \rightarrow \tau^{\prime}$. There are three cases to be considered: $0<\tau<\infty, \tau=0$ and $\tau=\infty$.

For the case $0<\tau<\infty$, since $h$ is a quasisymmetric homeomorphism, it follows that $0<\tau^{\prime}<\infty$. Thus, letting $n \rightarrow \infty$ in (7) yields a contradiction in this case.

To deal with the case $\tau=0$ or $\tau=\infty$, we observe that, by replacing $\tau_{n}$ by $1 / \tau_{n}$, we can assume that $a_{n}<c_{n}$. We shall use Lemma 1 to derive that

$$
\frac{1}{H_{h}} \leq \lim _{n \rightarrow \infty} \frac{\ln \tau_{n}}{\ln \tau_{n}^{\prime}} \leq H_{h}
$$

which immediately leads to a contradiction with (7). To this end, we need to consider three possible positions of $b_{n}$ relative to $a_{n}$ and $c_{n}$. First assume that $a_{n}<b_{n}<c_{n}$. Then (8) follows immediately from Lemma 1.

Next assume that $a_{n}<c_{n}<b_{n}$. Then

$$
\tau_{n}=\frac{\left|c_{n}-b_{n}\right|}{\left|b_{n}-a_{n}\right|}=\frac{1}{1+\sigma_{n}^{-1}},
$$

where

$$
\sigma_{n}=\frac{\left|b_{n}-c_{n}\right|}{\left|c_{n}-a_{n}\right|} \rightarrow 0
$$

since $1>\tau_{n} \rightarrow 0$. Thus it follows that

$$
\lim _{n \rightarrow \infty} \frac{\ln \tau_{n}}{\ln \tau_{n}^{\prime}}=\lim _{n \rightarrow \infty} \frac{\ln \sigma_{n}}{\ln \sigma_{n}^{\prime}} .
$$

Applying Lemma 1 to the sequences $a_{n}<c_{n}<b_{n}$ (and the corresponding $\sigma_{n}$ ), we obtain (8) as desired.

Finally, assume that $b_{n}<a_{n}<c_{n}$. Using a similar argument as above and applying Lemma 1 to the sequences $b_{n}<a_{n}<c_{n}$, one can derive inequalities (8). This completes the proof of Proposition 1.

The above argument can be modified to establish the following relation between the quasisymmetric exponent $\alpha_{h}$ and the dilatation $M_{h}$ for a quasisymmetric homeomorphism. This is somewhat surprising because $\alpha_{h}$ is a local constant while $M_{h}$ measures the global distortion of modulus by $h$.

Proposition 2. For any quasisymmetric homeomorphism $h$ of the real line $\mathbf{R}$ onto itself, $\alpha_{h} \leq M_{h}$ 
Proof. The idea and set up are the same as in the proof of Proposition 1. So we will use exactly the same notation as above and replace the boundary dilatation $H_{h}$ by the dilatation $M_{h}$ of a quasisymmetric homeomorphism. Thus, in the place of (7), we have

$$
\frac{1}{M_{h}+\varepsilon} \ln \frac{1}{\tau_{n}}-\ln \frac{1}{\tau_{n}^{\prime}} \rightarrow \infty \quad \text { and } \quad \ln \tau_{n}^{\prime}-\left(M_{h}+\varepsilon\right) \ln \tau_{n} \rightarrow \infty
$$

as $n \rightarrow \infty$.

Applying the inequalities for $M_{h}$ established in Lemma 1, the same argument as in the proof of Proposition 1 yields a contradiction with (9) as desired.

Combining Propositions 1 and 2, we obtain the following relationship among the four important conformal invariants $\alpha_{h}, M_{h}, H_{h}$ and $K_{h}$ of a homeomorphism.

Theorem 1. For any quasisymmetric homeomorphism $h$ of the real line $\mathbf{R}$ onto itself, we have

$$
\alpha_{h} \leq H_{h} \leq K_{h}, \alpha_{h} \leq M_{h} \leq K_{h} .
$$

These estimates will play a crucial role in establishing a necessary and sufficient condition for the equality $M_{h}=K_{h}$.

\section{A necessary and sufficient condition for $M_{h}=K_{h}$}

In this section we prove the following main result and derive some corollaries.

Theorem 2. Suppose $h$ is a quasisymmetric homeomorphism of the real line $\mathbf{R}$ onto itself. Then $M_{h}=K_{h}$ if and only if $\alpha_{h}=K_{h}$ or $h$ is induced by an affine mapping.

As pointed out in the introduction, the converse of Theorem A is not true, that is the equality $H_{h}=K_{h}$ (or existence of a substantial boundary point) is not sufficient to guarantee that $M_{h}=K_{h}$. By Theorem 2, however, if one replaces the boundary dilatation $H_{h}$ by the quasisymmetric exponent $\alpha_{h}$, then the condition in Theorem A becomes necessary and sufficient for the equality $M_{h}=K_{h}$.

4.1. Degenerate and non-degenerate cases for $M_{h}$. The proof of Theorem 2 involves delicate analysis on how the dilatation $M_{h}$ is attained. Before proceeding, we introduce the following terminology. Recall that, for a quasisymmetric homeomorphism $h$ of $\mathbf{R}$, the dilatation $M_{h}$ is defined as

$$
M_{h}=\sup \frac{\bmod (h(A), h(B) ; \mathbf{H})}{\bmod (A, B ; \mathbf{H})},
$$

where the supremum is taken over all pairs of disjoint nondegenerate continua $A$ and $B$ on $\overline{\mathbf{R}}$. Fix a sequence of pairs $A_{n}=\left[a_{n}, b_{n}\right]$ and $B_{n}=\left[c_{n}, d_{n}\right]$ of disjoint non-degenerate continua such that

$$
M_{h}=\lim _{n \rightarrow \infty} \frac{\bmod \left(h\left(A_{n}\right), h\left(B_{n}\right) ; \mathbf{H}\right)}{\bmod \left(A_{n}, B_{n} ; \mathbf{H}\right)} .
$$

By passing to subsequences, we may assume that $A_{n} \rightarrow A$ and $B_{n} \rightarrow B$. Depending on the sizes and positions of the limit sets $A$ and $B$, the following are the only possible ways in which $M_{h}$ can be attained.

We say that $M_{h}$ is attained by non-degenerate continua if the limit sets $A$ and $B$ are disjoint non-degenerate continua. In this case, by continuity of the modulus, 
there exist a pair of disjoint nondegenerate continua $A$ and $B$ on $\overline{\mathbf{R}}$ such that

$$
M_{h}=\frac{\bmod (h(A), h(B) ; \mathbf{H})}{\bmod (A, B ; \mathbf{H})} .
$$

We say that $M_{h}$ is attained by degenerate continua if one of the limit sets reduces to a point or $A \cap B \neq \emptyset$. In this case, there are sequences of points $a_{n} \rightarrow a, b_{n} \rightarrow b$, $c_{n} \rightarrow c, d_{n} \rightarrow d$ such that

$$
M_{h}=\lim _{n \rightarrow \infty} \frac{\bmod \left(\left[a_{n}^{\prime}, b_{n}^{\prime}\right],\left[c_{n}^{\prime}, d_{n}^{\prime}\right] ; \mathbf{H}\right)}{\bmod \left(\left[a_{n}, b_{n}\right],\left[c_{n}, d_{n}\right] ; \mathbf{H}\right)}
$$

and such that at least two of the limit points $a, b, c, d$ coincide, where $a_{n}^{\prime}, b_{n}^{\prime}, c_{n}^{\prime}, d_{n}^{\prime}$ are the images of $a_{n}, b_{n}, c_{n}, d_{n}$ under $h$. In the degenerate case, we say it is totally degenerate if the cross-ratios

$$
t_{n}=\left[a_{n}, b_{n}, c_{n}, d_{n}\right]=\frac{\left|c_{n}-b_{n}\right|\left|d_{n}-a_{n}\right|}{\left|b_{n}-a_{n}\right|\left|d_{n}-c_{n}\right|} \rightarrow 0 \text { or } \infty
$$

as $n \rightarrow \infty$. We say it is pseudo-degenerate if $t_{n}=\left[a_{n}, b_{n}, c_{n}, d_{n}\right] \rightarrow t \neq 0, \infty$ as $n \rightarrow \infty$. Note that these cases may or may not be mutually exclusive.

4.2. Proof of Theorem 2. For the sufficiency, if $\alpha_{h}=K_{h}$, it follows immediately from Theorem 1 that $M_{h}=K_{h}$. If $h$ is induced by an affine mapping, it is easy to see that $M_{h}=K_{h}$ as well because the affine map itself is an extremal QC extension of $h$.

For the proof of necessity in Theorem 2 , let $M_{h}=K_{h}$. We need to show that either $h$ is induced by an affine map or $\alpha_{h}=K_{h}$. This will be done by analyzing the three cases on how $M_{h}$ is attained: non-degenerate case, totally degenerate case, and pseudo-degenerate case as defined above. In the non-degenerate case and pseudodegenerate case, we shall show that $h$ is induced by an affine map. In the totally degenerate case, we derive that $M_{h} \leq \alpha_{h}$. This together with Theorem 1 and the equality $M_{h}=K_{h}$ yields that $\alpha_{h}=K_{h}$ as desired.

4.3. Non-degenerate case for $\boldsymbol{M}_{\boldsymbol{h}}$. In this case, $M_{h}$ is attained by nondegenerate continua, that is, there exist a pair of disjoint nondegenerate continua $A$ and $B$ on $\overline{\mathbf{R}}$ such that

$$
M_{h}=\frac{\bmod (h(A), h(B) ; \mathbf{H})}{\bmod (A, B ; \mathbf{H})} .
$$

Then, by the proof of Theorem A (see [26]), the equality $M_{h}=K_{h}$ implies that $h$ is induced by an affine map.

4.4. Reduction of degenerate case. To treat the totally degenerate case and pseudo-degenerate case efficiently, we first make a reduction on the general degenerate case for $M_{h}$. Assume that $M_{h}$ is attained by degenerate continua. Then there are sequences of points $a_{n} \rightarrow a, b_{n} \rightarrow b, c_{n} \rightarrow c, d_{n} \rightarrow d$ such that (10) holds and that at least two of the limit points $a, b, c, d$ coincide.

According to the possible positions of the limit points $a, b, c, d$, there are four degenerate cases to be considered:

(1) $a=b$ and $a, c, d$ distinct;

(2) $a=b, c=d$ and $a \neq c$;

(3) $a=b=c \neq d$

(4) $a=b=c=d$. 
However, due to the detailed analysis done in the proof of Theorem A in [26], one concludes that in all the degenerate cases one can choose sequences $a_{n}<b_{n}<c_{n}<$ $d_{n}$, all converging to the same point, say the origin, such that (10) holds. Thus, for the remainder of the proof, we assume that such sequences have been chosen.

4.5. Totally degenerate case. In this case, we have sequences $a_{n}<b_{n}<c_{n}<$ $d_{n}$, all converging to the origin, such that (10) holds and that

$$
t_{n}=\left[a_{n}, b_{n}, c_{n}, d_{n}\right]=\frac{\left|c_{n}-b_{n}\right|\left|d_{n}-a_{n}\right|}{\left|b_{n}-a_{n}\right|\left|d_{n}-c_{n}\right|} \rightarrow 0 \text { or } \infty .
$$

And let $t_{n}^{\prime}=\left[a_{n}^{\prime}, b_{n}^{\prime}, c_{n}^{\prime}, d_{n}^{\prime}\right]$.

First, assume $t_{n} \rightarrow \infty$. In this case, $t_{n}^{\prime} \rightarrow \infty$ as well due to the quasisymmetry of $h$. Thus, by Lemma 2 , it follows that

$$
M_{h}=\lim _{n \rightarrow \infty} \frac{\bmod \left(\left[a_{n}^{\prime}, b_{n}^{\prime}\right],\left[c_{n}^{\prime}, d_{n}^{\prime}\right] ; \mathbf{C}\right)}{\bmod \left(\left[a_{n}, b_{n}\right],\left[c_{n}, d_{n}\right] ; \mathbf{C}\right)}=\lim _{n \rightarrow \infty} \frac{\frac{2 \pi}{\ln \Psi\left(t_{n}^{\prime}\right)}}{\frac{2 \pi}{\ln \Psi\left(t_{n}\right)}}=\lim _{n \rightarrow \infty} \frac{\ln t_{n}}{\ln t_{n}^{\prime}} \leq \alpha_{h} .
$$

Hence in this case we have $M_{h} \leq \alpha_{h} \leq K_{h}$.

Next, assume $t_{n} \rightarrow 0$. By considering the conjugate quadrilateral of $Q\left(a_{n}, b_{n}, c_{n}\right.$, $\left.d_{n}\right)$, we obtain that

$$
M_{h}=\lim _{n \rightarrow \infty} \frac{1 / \bmod \left(\left[a_{n}, b_{n}\right],\left[c_{n}, d_{n}\right] ; \mathbf{C}\right)}{1 / \bmod \left(\left[a_{n}^{\prime}, b_{n}^{\prime}\right],\left[c_{n}^{\prime}, d_{n}^{\prime}\right] ; \mathbf{C}\right)}=\lim _{n \rightarrow \infty} \frac{\frac{2 \pi}{\ln \Psi\left(1 / t_{n}\right)}}{\frac{2 \pi}{\ln \Psi\left(1 / t_{n}^{\prime}\right)}} .
$$

Appealing to Lemma 2 again yields that

$$
M_{h}=\lim _{n \rightarrow \infty} \frac{\ln \left(1 / t_{n}^{\prime}\right)}{\ln \left(1 / t_{n}\right)} \leq \alpha_{h} \leq K_{h}
$$

Thus, in the totally degenerate case, we have $M_{h} \leq \alpha_{h} \leq K_{h}$. Therefore, the equality $M_{h}=K_{h}$ yields $\alpha_{h}=K_{h}$ as desired.

4.6. Pseudo-degenerate case. In this case, there exist sequences $a_{n}<b_{n}<$ $c_{n}<d_{n}$, all converging to the origin, such that (10) holds and that

$$
t_{n}=\left[a_{n}, b_{n}, c_{n}, d_{n}\right] \rightarrow t, \quad t_{n}^{\prime}=\left[a_{n}^{\prime}, b_{n}^{\prime}, c_{n}^{\prime}, d_{n}^{\prime}\right] \rightarrow t^{\prime}
$$

as $n \rightarrow \infty$, where the limits $t$ and $t^{\prime}$ are finite and positive. Thus it follows that

$$
M_{h}=\lim _{n \rightarrow \infty} \frac{\bmod \left(\left[a_{n}^{\prime}, b_{n}^{\prime}\right],\left[c_{n}^{\prime}, d_{n}^{\prime}\right] ; \mathbf{C}\right)}{\bmod \left(\left[a_{n}, b_{n}\right],\left[c_{n}, d_{n}\right] ; \mathbf{C}\right)}=\lim _{n \rightarrow \infty} \frac{\frac{2 \pi}{\ln \Psi\left(t_{n}^{\prime}\right)}}{\frac{2 \pi}{\ln \Psi\left(t_{n}\right)}}=\frac{\ln \Psi(t)}{\ln \Psi\left(t^{\prime}\right)} .
$$

We will use a compactness argument to show that there exists a quasisymmetric homeomorphism $g$ of $\mathbf{R}$ such that

$$
M_{g}=M_{h}, K_{g}=K_{h}
$$

and that $M_{g}$ is attained by non-degenerate continua.

For this we fix Möbius transformations $\varphi_{n}$ and $\psi_{n}$ such that

$$
\varphi_{n}\left(a_{n}\right)=-1, \varphi_{n}\left(b_{n}\right)=0, \varphi_{n}\left(c_{n}\right)=t_{n}, \varphi_{n}\left(d_{n}\right)=\infty
$$

and that

$$
\psi_{n}\left(a_{n}^{\prime}\right)=-1, \psi_{n}\left(b_{n}^{\prime}\right)=0, \psi_{n}\left(c_{n}^{\prime}\right)=t_{n}^{\prime}, \psi_{n}\left(d_{n}^{\prime}\right)=\infty .
$$

For $n=1,2, \ldots$, let

$$
g_{n}=\psi_{n} \circ h \circ \varphi_{n}^{-1} .
$$


Then $g_{n}$ fixes $-1,0$ and $\infty$, and $g_{n}\left(t_{n}\right)=t_{n}^{\prime}$. Furthermore, by the Möbius invariance of $M_{h}$ and $K_{h}$, it follows that

$$
M_{g_{n}}=M_{h} \quad \text { and } \quad K_{g_{n}}=K_{h}
$$

for any $n \geq 1$. Also we have $H_{g_{n}}=H_{h}$. Next, let $f_{n}$ be an extremal quasiconformal extension of $g_{n}$ to $\mathbf{C}$. Due to the compactness of the family $\left\{f_{n}\right\}$, we conclude (by passing to a subsequence if necessary) that $f_{n}$ converges uniformly (in the spherical metric) to a quasiconformal mapping $f$. Denote the restriction of $f$ to the real line by $g$. Then, $g$ also fixes $-1,0, \infty$ and $g(t)=t^{\prime}$. Moreover, it follows from the uniform convergence that

$$
M_{g}=\lim _{n \rightarrow \infty} M_{g_{n}}=M_{h}, \quad K_{g}=K_{h} .
$$

This yields that $M_{g}$ is attained by the non-degenerate continua $[-1,0]$ and $[t, \infty]$.

Now assume that the equality $M_{h}=K_{h}$ holds. Then we have $M_{g}=K_{g}$. Applying the non-degenerate case treated above to the quasisymmetric homeomorphism $g$, we conclude that $g$ is induced by an affine map. Hence $H_{g}<K_{g}$. This means that $g$ is a Strebel point in the universal Teichmüller space (see, for example, [13]). Furthermore, since the set of Strebel points is open in the universal Teichmüller space (see [13]) and $g_{n}=\psi_{n} \circ h \circ \varphi_{n}^{-1} \rightarrow g$, it follows that $g_{n}$ (for large $n$ ), and hence $h$, is a Strebel point as well. Thus, $h$ does not have a substantial boundary point. Therefore, by Theorem A, the equality $M_{h}=K_{h}$ implies that $h$ is induced by an affine map. This completes the proof of Theorem 2.

4.7. Remark. The above proof shows that, in the totally degenerate case where the cross ratio $t_{n}=\left[a_{n}, b_{n}, c_{n}, d_{n}\right]$ converges to 0 or $\infty$, we have $M_{h} \leq \alpha_{h}$. Combining this with Proposition 2, it follows that $M_{h}=\alpha_{h}$. This reveals an intimate relation between the dilatation $M_{h}$ and quasisymmetric exponent $\alpha_{h}$ of a homeomorphism $h$.

4.8. Corollaries. We conclude this section by deriving several corollaries from the above results. Combining Theorem 2 and the proof of Theorem B (or its degenerate case considered in [6]), we obtain the following equivalent conditions for the non-trivial case when $h$ is not induced by an affine map.

Corollary 1. Suppose $h$ is a quasisymmetric homeomorphism of the real line $\mathbf{R}$ onto itself. If $h$ is not induced by affine mapping, then the following conditions are all equivalent:

(1) $\alpha_{h}=K_{h}$.

(2) $M_{h}=K_{h}$.

(3) There exists an extremal quasiconformal extension of $h$ whose complex dilatation $\mu$ satisfies

$$
\lim _{n \rightarrow \infty} \frac{\operatorname{Re} \iint_{\mathbf{H}} \mu(z) \Phi_{Q_{n}}^{\prime 2}(z) d x d y}{\iint_{\mathbf{H}}\left|\mu(z) \Phi_{Q_{n}}^{\prime 2}(z)\right| d x d y}=\|\mu\|_{\infty}
$$

where $\Phi_{Q_{n}}$ maps a degenerating topological quadrilateral sequence $Q_{n}=$ $Q\left(z_{1}^{n}, z_{2}^{n}, z_{3}^{n}, z_{4}^{n}\right)$ conformally onto a rectangle

$$
R_{n}=\left\{\zeta=\xi+i \eta: 0<\xi<1,0<\eta<b_{n}\right\} .
$$

Next, we illustrate how the above results can be used to determine whether the two dilatations $M_{h}$ and $K_{h}$ are the same for a given homeomorphism $h$. First we consider the case when $\alpha_{h}=1$. The following Corollary can be derived easily from Theorem 2. 
Corollary 2. Let $h$ be a quasisymmetric homeomorphism of the real line $\mathbf{R}$ onto itself which is not Möbius and not induced by an affine map. If $\alpha_{h}=1$, then $M_{h}<K_{h}$

This corollary looks simple. But it can be applied to a variety of examples. One of them is the following well known Strebel example:

$$
h(x)= \begin{cases}K x, & x \geq 0 \\ x, & x<0\end{cases}
$$

for some $K>1$. It is shown that (see $[16,21]$ )

$$
f(z)=K^{1-\frac{1}{\pi} \arg z} z
$$

is an extremal quasiconformal extension of $h$ onto $\mathbf{H}$ and that

$$
H_{h}=K_{h}=1+\frac{1}{2 \pi^{2}} \ln ^{2} K+\frac{1}{\pi} \ln K \sqrt{1+\frac{1}{4 \pi^{2} \ln ^{2} K}} .
$$

Using these and some sophisticated calculation, Shen [16] showed that $M_{h}<K_{h}$ for this $h$. On the other hand, it is easy to see that the quasisymmetric exponent of the above $h$ is $\alpha_{h}=1$. Thus it follows immediately from Theorem 2 (or the above corollary) that $M_{h}<K_{h}$.

For a wider class of examples, we introduce the concept of locally linear homeomorphism.

Definition 2. A homeomorphism $h$ of $\mathbf{R}$ onto itself is said to be locally linear if for any $x \in \mathbf{R}$, there exist a left side neighborhood $U^{-}(x)$ and right side neighborhood $U^{+}(x)$, such that $h$ is a linear function in both $U^{-}(x)$ and $U^{+}(x)$.

Apparently, any piecewise linear homeomorphism is locally linear. In particular, Strebel's example is locally linear. It is easy to see that for a locally linear homeomorphism $h$, we always have $\alpha_{h}=1$. Thus the following result follows.

Corollary 3. If $h$ is a locally linear homeomorphism other than a Möbius map, then $M_{h}<K_{h}$.

Finally, we consider the other end of the spectrum for $\alpha_{h}: \alpha_{h}=K_{h}$. The following result follows directly from Proposition 1.

Corollary 4. If $\alpha_{h}=K_{h}$, then $h$ has a substantial boundary point.

However, as illustrated by the above Strebel example, the converse of this result is not true. It shows that substantial boundary points can occur even when $\alpha_{h}=1$.

\section{Applications}

We conclude this paper with two more applications of the above results. One is an attempt to classify elements in the universal Teichmüller space. The other is to estimate some domain constants.

5.1. Classification of quasisymmetric homeomorphisms. Consider the set of all quasisymmetric homeomorphisms of $\mathbf{R}$ onto itself. Two homeomorphisms $h_{1}$ and $h_{2}$ are called equivalent if there exists some conformal automorphism $\phi$ of the extended complex plane $\overline{\mathbf{C}}$ such that $h_{1}=h_{2} \circ \phi$. The set of equivalent classes is known as the universal Bers' Teichmüller space $T$ (see, for example, [14]). By Earle and Li [7], a quasisymmetric homeomorphism (or its equivalence class) in the universal Teichmüller space is called a Strebel point if $H_{h}<K_{h}$. Following a result 
of Lakic [13], the set of Strebel points is open and dense in the universal Teichmüller space $T$. It is obvious that a quasisymmetric homeomorphism $h$ is not a Strebel point if and only if it has a substantial boundary point. It is also well known that (see, for example, $[19,20]$ ) a quasisymmetric homeomorphism $h$ induced by an affine mapping is a Strebel point.

A classification of all quasisymmetric homeomorphisms can be obtained by using the above results. By Theorem 1 and Theorem 2, if $\alpha_{h}=K_{h}$, then all the four constants $\alpha_{h}, H_{h}, M_{h}$ and $K_{h}$ are equal. We call a quasisymmetric homeomorphism having this property an essential quasisymmetric homeomorphism. The following result follows easily from above discussion.

Theorem 3. Any quasisymmetric homeomorphism $h$ of the real line $\mathbf{R}$ onto itself belongs to one and only one of the following classes.

(1) $\alpha_{h}=K_{h}$ (that is, $h$ is an essential quasisymmetric homeomorphism);

(2) $\alpha_{h}<K_{h}$ :

(2.1) $H_{h}=K_{h}$ ( $h$ has a substantial boundary point).

(2.2) $H_{h}<K_{h}$ ( $h$ is a Strebel point).

Since $H_{h}<K_{h}=M_{h}$ for any affine map, a quasisymmetric homeomorphism induced by an affine map is not an essential quasisymmetric homeomorphism and it belongs to class (2.2). While the Strebel's example belongs to class (2.1), an essential quasisymmetric homeomorphism is given by the following example. For any $\alpha \geq 1$, let

$$
h(x)= \begin{cases}x^{\alpha}, & x \geq 0 \\ -|x|^{\alpha}, & x<0\end{cases}
$$

Then $h$ is a quasisymmetric homeomorphism with quasisymmetric exponent $\alpha_{h}=\alpha$. Note that $h$ is the boundary value of the quasiconformal map $f(z)=|z|^{\alpha-1} z$. Thus $K_{h}=\alpha$ and $h$ is an essential quasisymmetric homeomorphism.

5.2. QED constants. For a Jordan domain $\Omega$ in the complex plane, consider the following quasiextremal distance (or QED) constant introduced by Yang [27]:

$$
M(\Omega)=\sup \frac{\bmod (A, B ; \mathbf{C})}{\bmod (A, B ; \Omega)}
$$

where the supremum is taken over all pairs of disjoint continua $A$ and $B$ in $\bar{\Omega}$ such that $\bmod (A, B ; \mathbf{C})$ and $\bmod (A, B ; \Omega)$ are not simultaneously zero or infinity. A domain $\Omega$ is called a QED domain if its QED constant $M(\Omega)$ is finite. QED domains were first introduced by Gehring and Martio (see [9] ) in connection with the theory of quasiconformal mappings, and later studied by many others (see [12, 27], etc). Quasi-extremal distance constant reflects the geometric properties of domain $\Omega$ and measures how far $\Omega$ is from being a disk. It was proved in [9] that a finitely connected domain $\Omega$ is a QED domain if and only if $\Omega$ is a quasicircle domain.

There are two other closely related domain constants. One is the quasiconformal reflection constant, define as

$$
R(\Omega)=\inf \{K(f): f \text { is a quasiconformal reflection in } \partial \Omega\} .
$$

The other, called the boundary QED constant, is defined as

$$
M_{b}(\Omega)=\sup \left\{\frac{\bmod (A, B ; \mathbf{C})}{\bmod (A, B ; \Omega)} \text { : for all pairs } A \text { and } B \text { in } \partial \Omega\right\} .
$$


It is well known that [27]

$$
2 \leq M_{b}(\Omega) \leq M(\Omega) \leq 1+R(\Omega) .
$$

The question of when the above equalities hold has attracted many authors' attention (see, for example, [10, 17, 25, 28, 29]). The connection between these domain constants and the conformal invariants of a homeomorphism studied above is established through a homeomorphism induced by a Jordan domain.

For a Jordan domain $\Omega$ in the extended complex plane $\overline{\mathbf{C}}$, let $f_{1}$ and $f_{2}$ map $\Omega$ and $\Omega^{*}=\overline{\mathbf{C}} \backslash \bar{\Omega}$ conformally onto upper half plane $\mathbf{H}$ and lower half plane $\mathbf{H}^{*}$, respectively. Extending $f_{1}$ and $f_{2}$ to the boundary $\partial \Omega$ and $\partial \Omega^{*}$, one can define $h_{\Omega}=\left.f_{2} \circ f_{1}^{-1}\right|_{\mathbf{R}}$ as the sewing mapping of the domains $\Omega$ and $\Omega^{*}$. We call $h_{\Omega}$ a homeomorphism induced by $\Omega$. It is easy to see that

$$
R(\Omega)=R\left(\Omega^{*}\right)=K_{h_{\Omega}}
$$

and

$$
M_{b}(\Omega) \geq 1+M_{h_{\Omega}} .
$$

Combining this with Theorem 2, we obtain the following sufficient condition for $M_{b}(\Omega)=M(\Omega)$ and $M(\Omega)=1+R(\Omega)$.

Theorem 4. Let $h_{\Omega}$ be a homeomorphism of $\mathbf{R}$ induced by a Jordan domain $\Omega$. Then $M_{b}(\Omega)=M(\Omega)=1+R(\Omega)$ if $\alpha_{h_{\Omega}}=K_{h_{\Omega}}$.

However, whether the condition is necessary remains open.

Acknowledgement. This work was partially done during two academic visits. One was done by the first author to the Department of Mathematics and Computer Science at Emory University. The other was done by the second author to the Department of Mathematics at East China Normal University. They wish to express their gratitude to both host institutions for their hospitality and support. Finally, they would also like to thank the referee for his/her valuable suggestions on the reorganization of the materials, especially in Section 3.

\section{References}

[1] Ahlfors, L. V.: Lectures on quasiconformal mappings. - Van Nostrand Mathematical Studies 10, D. Van Nostrand Co. Inc., Toronto-New York-London, 1966.

[2] Ahlfors, L. V.: Conformal invariants. - McGraw-Hill, New York, 1973.

[3] Anderson, J., and A. HinkKanen: Quadrilaterals and extremal quasiconformal extensions. - Comment. Math. Helv. 70, 1995, 455-474.

[4] Beurling, A., and L. V. Ahlfors: The boundary correspondence under quasiconformal mappings. - Acta Math. 96, 1956, 125-142.

[5] Chen, J., and Z. Chen: A remark on "an approximation condition and extremal quasiconformal extension". - Chinese Science Bulletin 42, 1997, 1765-1767.

[6] Chen, Z., X. Zheng, and G. YaO: Quadrilaterals, extremal quasiconformal extensions and Hamilton sequences. - Appl. Math. J. Chinese Univ. 25, 2010, 217-226.

[7] EARLE, C. J., and Z. Li: Isometrically embedded polydisks in infinite dimensional Teichmüller space. - J. Geom. Anal. 9, 1999, 51-71.

[8] Fehlmann, R.: Qusaiconformal mappings with free boundary components. - Ann. Acad. Sci. Fenn. Math. 6, 1986, 323-335.

[9] Gehring, F. W., and O. Martio: Quasiextremal distance domains and extension of quasiconformal mappings. - J. Anal. Math. 45, 1985, 181-206. 
[10] Garnett, J. B., and S. Yang: Quasiextremal distance domains and integrability of derivatives of conformal mappings. - Michigan Math. J. 41, 1994, 389-406.

[11] Heinonen, J.: Lectures on analysis on metric spaces. - Spring-Verlag, New York, 2001.

[12] Herron, D. A., and P. Koskela: Quasiextremal distance domains and conformal mappings onto circle domains. - Complex Variables 15, 1990, 167-179.

[13] Lakic, N.: Strebel points. - Contemp. Math. 211, 1997, 417-431.

[14] Leнto, O.: Univalent functions and Teichmüller spaces. - Springer-Verlag, New York, 1986.

[15] Reich, E., and K. Strebel: Extremal quasiconformal mappings with given boundary values. - In: Contributions to analysis (a collection of papers dedicated to Lipman Bers), Academic Press, New York, 1974, 375-391.

[16] Shen, Y.: A counterexample theorem in quasiconformal mapping theory. - Sci. China Ser. A. 43, 2000, 929-936.

[17] Shen, Y.: Conformal invariants of QED domains. - Tohoku Math. J. 56, 2004, 445-466.

[18] Shiga, H., and H. Tanigawa: Grunsky's inequality and its applications to Teichmüller space. - Kodai Math. J. 16, 1993, 361-378.

[19] Strebel, K.: On the dilatation of extremal quasiconformal mappings of polygons. - Comment. Math. Helv. 74, 1999, 143-149.

[20] Strebel, K.: Extremal quasiconformal mappings. - Results Math. 10, 1986, 169-209.

[21] Strebel, K.: Zur Frage de Eindeutigkeit extremaler quasikonformer Abbikdungen des Einheitskreises. - Comment. Math. Helv. 36, 1962, 306-323.

[22] Strebel, K.: On the existence of extremal Teichmüller mappings. - J. Anal. Math. 30, 1976, 464-480.

[23] Tukia, P., and J. VÄIsÄLÄ: Quasisymmetric embeddings of metric spaces. - Ann. Acad. Sci. Fenn. Math. 5, 1980, 97-114.

[24] Wu, S.: Moduli of quadrilaterals and extremal quasiconformal extensions of quasisymmetric functions. - Comment. Math. Helv. 72, 1997, 593-604.

[25] Wu, S., and S. Yang: On symmetric quasicircles. - J. Austr. Math. Soc. Ser. A 68, 2000, $131-144$.

[26] Yang, S.: On dilatations and substantial boundary points of homeomorphisms of Jordan curves. - Results Math. 31, 1997, 180-188.

[27] YAng, S.: QED domains and NED sets in $\overline{\mathbf{R}}^{n}$. - Trans. Amer. Math. Soc. 334, 1992, 97-120.

[28] YANG, S.: Conformal invariants of smooth domains and extremal quasiconformal mappings of ellipses. - Illinois J. Math. 41, 1997, 438-452.

[29] YANG, S.: A modulus inequality for condensers and conformal invariants of smooth domains. - J. Anal. Math. 75, 1998, 173-183.

Received 6 February 2015 • Accepted 28 August 2015 